Results The length of the reports ranged from 11-210 pages (average 86). A definitive purpose for the inquiry was stated in $6 / 10$ reports. Half of the reports explained the inquiry methodology used (including reference to review tools).

The inquiry team was named and multi-disciplinary (MDT) in five reports (4-14 people); four reports described each person's role, but not their responsibilities. It was clearly stated that affected families were involved in four inquiries and relevant clinical staff in four. The inquiry team commented on good aspects of care provided by clinical staff in only four reports.

In the recommendations four main domains were identified: management of information; pregnancy loss care; maternity services governance; workforce staffing and training. The SMART principle (i.e. specific, measurable, achievable, realistic or time-bound) was not consistently applied to the recommendations in any report, thereby reducing the chance of implementation.

Conclusion This was the first structured review of pregnancyloss national inquiry reports, highlighting some of the main issues arising from them. The methodology used during the inquiry process was clearly described in only half of reports. Clearly standardising inquiry processes and highlighting all relevant issues is essential. For this purpose, structured national report checklists can be a useful resource. Additionally, a selected external expert MDT, clinical staff and families should always be involved in the inquiry process. Recommendations made within inquiry reports, can have a profound impact on maternity services if implemented appropriately. This will be explored further in an analysis of all implemented recommendations.

\section{P21 PATHWAYS TO ETHNIC DIFFERENCES IN OBESITY: THE ROLE OF MIGRATION, CULTURE AND SOCIO-ECONOMIC POSITION IN THE UK}

V Higgins*, J Nazroo, M Brown. Cathie Marsh Institute for Social Research, University of Manchester, Manchester, UK

\subsection{6/jech-2019-SSMabstracts. 172}

Background Previous research has identified ethnic differences in abdominal obesity but has not fully explored the pathways that explain these ethnic differences, which may relate to individual and contextual characteristics. This research identifies ethnic differences in waist circumference for eight ethnic groups in England, before and after accounting for a range of individual-level and area-level factors. Three key pathways to obesity are explored: migration, culture, and socio-economic characteristics.

Methods Data come from four years of the Health Survey for England (1998, 1999, 2003 and 2004) and linked area-level data from the 2001 Census. The total sample size is 27,946 . Multi-level modelling methods are used to account for individual-level and area-level factors. The main outcome measure is objectively measured waist circumference. Area-level variables are Index of Multiple Deprivation and Co-ethnic density.

Results The results show that migration to the UK has a strong association with ethnic differences in waist circumference - in particular, waist circumference increases with length of time since migration to the UK. Cultural characteristics and socio-economic characteristics are also associated with ethnic differences in waist circumference, but not to the same extent as migration. The strong association between migration and waist circumference is partly attenuated by cultural characteristics and partly by socio-economic inequality. However, there is still a large association between migrant status and waist circumference that remains unexplained. Area deprivation has an association with ethnic differences in obesity.

Conclusion This research makes an important contribution to the study of ethnic differences in waist circumference in England. It is the first UK study to explore the associations of migration, cultural and socio-economic pathways with ethnic differences in waist circumference for a wide range of ethnic groups, using both individual and contextual data. The resultsillustrate the multi-dimensional nature of ethnic inequalities in obesity and the complex interplay between migration, culture and socio-economic inequality. Policy makers should focus on obesity prevention among child and adult migrants, particularly focussing on length of time since migration, the removal of barriers to educational attainment and employment among migrant populations and the effect of the areas that migrants live, particularly in relation to area deprivation. Obesity is an important factor in many health-related outcomes so the regular tracking of ethnic inequalities in obesity and a clear understanding of the pathways behind these differences is fundamental for improving the health of ethnic minority people.

\section{P22 USING NATIONAL HOSPITALISATION DATA TO EXPLAIN TRENDS IN INPATIENT ADMISSIONS FOR COPD IN IRELAND}

${ }^{1}$ E Hurley ${ }^{*},{ }^{2} \mathrm{~S}$-J Sinnott, ${ }^{3} \mathrm{~J}$ Browne, ${ }^{4} \mathrm{~T} \mathrm{MCDonnell,}{ }^{5} \mathrm{M}$ O'Connor, ${ }^{1} \mathrm{C}$ Normand. ${ }^{1} \mathrm{C}$ entre for Health Policy and Management, Trinity College Dublin, Ireland; ${ }^{2}$ Department of Noncommunicable Disease Epidemiology, London School of Hygiene and Tropical Medicine, UKi ${ }^{3}$ School of Public Health, University College Cork, Cork, Ireland; ${ }^{4}$ School of Medicine, University College Dublin, Ireland; ${ }^{5}$ Department of Public Health, Health Services Executive, Dublin, Ireland

\subsection{6/jech-2019-SSMabstracts.173}

Background Ireland has the highest rates of hospitalisations for Chronic Obstructive Pulmonary Disease (COPD) of all OECD countries $(369 / 100,000$ in 2017 , compared with OECD average of 200/100,000). We analysed national hospitalisation data to investigate the characteristics of these hospitalisations and how they have changed over time to assist in programme planning and identify areas for intervention.

Methods Using Hospital In-Patient Enquiry (HIPE), a national discharge database for episodes of admitted care in acute public hospitals, we undertook a longitudinal review of trends in inpatient hospitalisations for COPD between 2009 and 2017. COPD hospitalisations were identifiable by ICD-10-AM codes J40-J44 and J47. We standardised against two populations: 1) sex-specific rates against the OECD pop and 2) age-sex rates to the Irish population for adults aged 15 years and over

Results The prevalence of overnight hospitalisations increased for females from an age-standardised rate of 367.5/100,000 in 2009 to $393.0 / 100,000$ in 2017, and declined for males 391.6/100,000 to 343.5/100,000. Between 2009 and 2017, age-specific rates in males declined across most age categories, with the decline most pronounced in the 80-84 year age group. In contrast, females saw an increase in rates across the majority of age categories.

In 2017, rates were significantly higher amongst women in each 5 - year age category $\leq 70$ years, apart from the 55-59 
year category, where there was no difference. Rates were higher amongst men in each of the three age categories $\geq 75$ years. Our analysis indicates that the disparity between hospitalisations for older men and women has narrowed over the period of our study, owing to increased hospitalisations in women and decreased hospitalisation in men.

Conclusion Our analysis of hospitalisation data has seen a convergence in rates between the sexes and a worrying rise in hospitalisations amongst women, particularly among those less than 70 years. This mirrors trends internationally where women have higher rates of COPD than men throughout most of their lifespan, although it appears that they are especially vulnerable before the age of 65 . It is known that women are more susceptible than men to lung damage from cigarette smoke and other pollutants, and recent evidence highlights that they have more severe COPD exacerbations than males, resulting in higher hospitalisations. This study confirms these trends and points to the urgent need to raise awareness of this preventable disease, and tackle the high smoking rates that still prevail in Ireland, particularly in socioeconomically deprived areas.

\section{P23 USING NATIONAL PHARMACY CLAIMS DATA TO ESTIMATE THE PREVALENCE AND PATTERNS OF MEDICATION USE FOR COPD IN IRELAND}

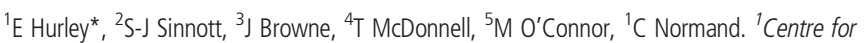
Health Policy and Management, Trinity College Dublin, Dublin, Ireland; ${ }^{2}$ Department of Non-communicable Disease Epidemiology, London School of Hygiene and Tropical Medicine, London, UK; ${ }^{3}$ School of Public Health, University College Cork, Cork, Ireland; ${ }^{4}$ School of Medicine, University College Dublin, Dublin, Ireland; ${ }^{5}$ Department of Public Health, Health Services Executive, Dublin, Ireland

\subsection{6/jech-2019-SSMabstracts. 174}

Background Ireland's National Clinical Programmme recently produced clinical guidelines on pharmacotherapy for COPD. However, there is no coherent picture of the prevalence of COPD in Ireland, the characteristics of who is receiving medication for COPD and how prescribing aligns with best practice. In view of this, we evaluated medication dispensing data to estimate the age and sex specific prevalence of symptomatic COPD in Ireland, and to identify patterns of medication use which can be used to inform interventions to improve the management of this condition.

Methods We used the Primary Care Reimbursement Service database, a national repository of anonymised pharmacy claims for more than $40 \%$ of the Irish population who qualify for the General Medical Services (GMS) scheme; a public health insurance programme for those of lower income and those $\geq 70$ years. We used a cohort study design with data from 2016 , limiting our population to those aged $\geq 45$ years to help remove dispensing for asthma. We examined the distribution of all respiratory medications dispensed, and then the patterns of medication use in those likely to have COPD.

Results From the GMS eligible population (aged $\geq 45$ years) with coverage for the entire year $(n=730,832)$, there were 170,950 patients dispensed at least one respiratory medication in 2016; equating to approximately $23 \%$ of the GMS population $\geq 45$ years receiving at least one respiratory medication (21.5\% of males and $25.0 \%$ of females).

The prevalence of medication use suggestive of COPD in those aged $\geq 45$ years was $15.1 \%$ (m) and $16.2 \%$ (f). Prevalence was higher in females than males aged between 45 and
64 years $(13.1 \%$ vs. $10.1 \%, \mathrm{p}<0.0001)$; there was no gender difference in those aged 65-74 years; and prevalence was higher in males aged $\geq 75$ years $(21.7 \%$ vs. $18.9 \%$, $\mathrm{p}<0.0001)$. Prevalence peaked in both sexes in the oldest age category: $21.7 \%$ (m) and $18.9 \%$ (f). There was significant inhaled corticosteroid (ICS) use, along with underuse of LAMA therapy.

Conclusion The prevalence of medication use consistent with the management of symptomatic COPD mirrors international estimates on prevalence of COPD, and in the absence of a population-based prevalence study, can be used to inform decision making. The high use of ICS, and the under use of LAMA therapy and poor adherence of those newly initiated is of concern. We recommend the development of an educational intervention for health professionals to assist in the implementation of new national prescribing guidelines for the management of COPD.

\section{P24 ONLINE DATING AND SEXUALLY TRANSMITTED INFECTIONS IN ENGLAND: AN ECOLOGICAL STUDY USING GOOGLE TRENDS DATA}

$1,{ }^{2}$ LKRP Jennings* ${ }^{2} \mathrm{C}$ Kypridemos. ${ }^{1}$ School of Public Health, Health Education England
North West, Greater Manchester, UK; ${ }^{2}$ Department of Public Health and Policy, University of
Liverpool, Liverpool, UK

10.1136/jech-2019-SSMabstracts. 175

Background Online dating, and the use of dating smartphone applications has been theorised as a driver behind recent increases in some sexually transmitted infections (STIs). Existing literature is mixed, but some studies demonstrate an increase in high-risk sexual behaviours or STIs in online daters. However, many previous studies have a narrow study population and often rely on survey or interview data, which may not be representative. This ecological study aimed to investigate the association between online dating and STIs by using existing aggregated population-level data.

Methods We used official Public Health England STI incidence data by county or unitary authority in England for 2017. Based on Google Trends internet search data for the names of frequently used dating applications, we constructed a proxy measure for dating application usage, by English location in 2017. We named this measure 'proxy dating app score'. We used generalised linear modelling to explore the association between STI incidence and ranked proxy dating app score while controlling for known confounders (area index of multiple deprivation, proportion of people of black ethnicity in the population, and proportion of people aged under 25 in the population), assuming multiplicative effects. We performed extensive sensitivity analysis to test our assumptions, including a binomial regression model with STI incidence rates as the dependent variable. We used SPSS v22 to conduct the analysis.

Results In our main analysis, we observed a positive association between STI incidence and ranked proxy dating app score. Every increase in proxy dating app score rank was linked to a $7 \%(95 \% \mathrm{CI}: 4 \%$ to $11 \%, \mathrm{p}<0.01)$ rise in the count of STIs. Area deprivation and proportion of people of black ethnicity were also positively associated with count of STIs, $19 \%$ (95\% CI: $13 \%$ to $25 \%$ ) and $23 \%$ (95\% CI: $20 \%$ to $27 \%$ ) respectively, while the proportion of people aged under 25 was not a significant predictor. The direction of the associations did not change in the sensitivity analysis. 